\title{
IN UN MONDO NEL MONDO
}

Maria Spalletta ${ }^{77}$

\begin{abstract}
Sintesi
In questo articolo presento la mia personale esperienza di Service Learning Internazionale, sviluppata attraverso il progetto INTEREURISLAND come tirocinio prelaurea del corso in Psicologia dello Sviluppo, della Personalità e delle Differenze Individuali presso l'Università degli studi di Padova. Nello specifico riporto le esperienze formative realizzate attraverso: le diverse attività con gli adolescenti in conflitto con la legge accolti presso il CASE Gey Espinheira, struttura rieducativa di semilibertà della città di Juazeiro-BA (BR); la frequentazione del corso di "Dinamiche di Gruppo e Relazioni Interpersonali" al Dipartimento di Scienze Umane, Campus III / dell'Università dello Stato della Bahia / UNEB; la partecipazione a due eventi all'insegna del dialogo interculturale quali il I Colóquio Brasil Itália e l'ENINEPE. Questo articolo ha come obiettivo il favorire e promuovere la mobilità studentesca e giovanile attraverso esperienze formative interculturali che si dimostrano essere fonti di grandi ricchezze e occasioni di riscoperta.
\end{abstract}

Parole chiave: INTEREURISLAND. Intercultura. Adolescenti in conflitto con la legge. Tirocinio all'estero. Service Learning Internazionale.

\section{EM UM MUNDO NO MUNDO}

\section{Resumo}

Neste artigo, apresento a minha experiência pessoal de Aprendizagem Solidária International, desenvolvida através do projeto INTEREURISLAND como estágio do curso de graduação em Psicologia do Desenvolvimento, Personalidade e Diferenças Individuais na Universidade de Pádua. Relato especificamente as experiências formativas realizadas nas diversas atividades com adolescentes em conflito com a lei acolhidos no CASE Gey Espinheira, uma estrutura ré educacional de semiliberdade na cidade de Juazeiro-BA (BR), a participação no curso de 'Dinâmica de Grupos e Relações Interpessoais' no Departamento de Ciências Humanas, Campus III / Universidade da Bahia / UNEB e a participação em dois eventos dedicados ao diálogo intercultural: o I Colóquio Brasil Itália e a ENINEPE. Este artigo tem como objetivo incentivar e promover a mobilidade internacional de estudantes e jovens por meio de experiências de formação intercultural que provam ser fontes de grande riqueza e oportunidades de redescoberta.

\footnotetext{
77 Studentessa del corso di laurea Psicologia dello Sviluppo, della Personalità e delle Differenze Individuali presso 1’Università degli studi di Padova mary.spalletta.ms@gmail.com
} 
Palavras-chave: INTEREURISLAND. Intercultura. Adolescentes em conflito com a lei. Estágio no exterior. Aprendizagem Solidária Internacional.

\title{
IN A WORLD IN THE WORLD
}

\begin{abstract}
In this article I present my personal experience of International Service Learning, developed through the INTEREURISLAND project as a pre-graduate internship of the course in Developmental Psychology, Personality and Individual Differences at the University of Padua. Specifically, I report the educational experiences carried out through: the various activities with adolescents in conflict with the law, under semifreedom social-educative measure, hosted at the CASE Gey Espinheira institution, in the city of Juazeiro-BA (BR); the attendance of the course on 'Interpersonal relationships and group dynamics' at the Department of Human Sciences, Campus III / University of the State of Bahia / UNEB; participation in two events dedicated to intercultural dialogue such us I Colóquio Brasil Itália and ENINEPE. This article aims to encourage and promote student and youth mobility through intercultural educational experiences that prove to be sources of great wealth and opportunities for rediscovery.
\end{abstract}

Keywords: INTEREURISLAND. Interculture. Teenagers in conflict with the law. Internship abroad. International Service Learning.

Tra i mesi di agosto ed ottobre del 2019 ho svolto in Brasile il mio tirocinio prelaurea del corso triennale in Psicologia dello Sviluppo, della Personalità e delle Relazioni Interpersonali del Dipartimento di Psicologia dello Sviluppo e della Socializzazione DPSS, dell'Università degli studi di Padova/ UNIPD. Tra le varie opportunità proposte del mio Ateneo, ho deciso di partecipare al progetto di ricerca e scambi interculturali INTEREURISLAND, coordinato da Nicola Andrian, ricercatore vincolato al programma Post Lauream 'Mestrado' in Educação, Cultura e Territórios Semiáridos - PPGESA ${ }^{78}$, al Dipartimento di Scienze Umane DCH, Campus III dell'Università dello Stato della Bahia /UNEB nella regione nord-est del Brasile. Questo progetto si sviluppa attraverso la proposta pedagogica del Service-Learning, con un approccio internazionale, proponendo l'alternanza tra attività di studio al DCH III / UNEB e attività pratiche di tirocinio in alcune strutture partner del territorio di Juazeiro-BA.

\footnotetext{
${ }^{78}$ Mia traduzione: Educazione Cultura e Territori Semiaridi.
} 
Questa esperienza di formazione è stata molto di più di un semplice viaggio di studio, direi un viaggio dentro me stessa e dentro un nuovo modo di osservare, sentire e comunicare. In accordo con il piano di mobilità internazionale del progetto (come presentato in Figura 1 di seguito), nella fase di formazione pre-partenza, svolta da marzo a giugno del 2019, ho partecipato ad un percorso specifico di formazione che ha previsto diversi incontri sulla lingua e sulla cultura brasiliane (tenuti dalla studentessa brasiliana Jamile Soares da Silva del corso di laurea in Pedagogia del DCH III /UNEB, che era in mobilità a Padova proprio attraverso lo scambio INTEREURISLAND) e ad un weekend formativo residenziale in bivacco.

Figura 1 - Piano di mobilità internazionale studenti INTEREURISLAND

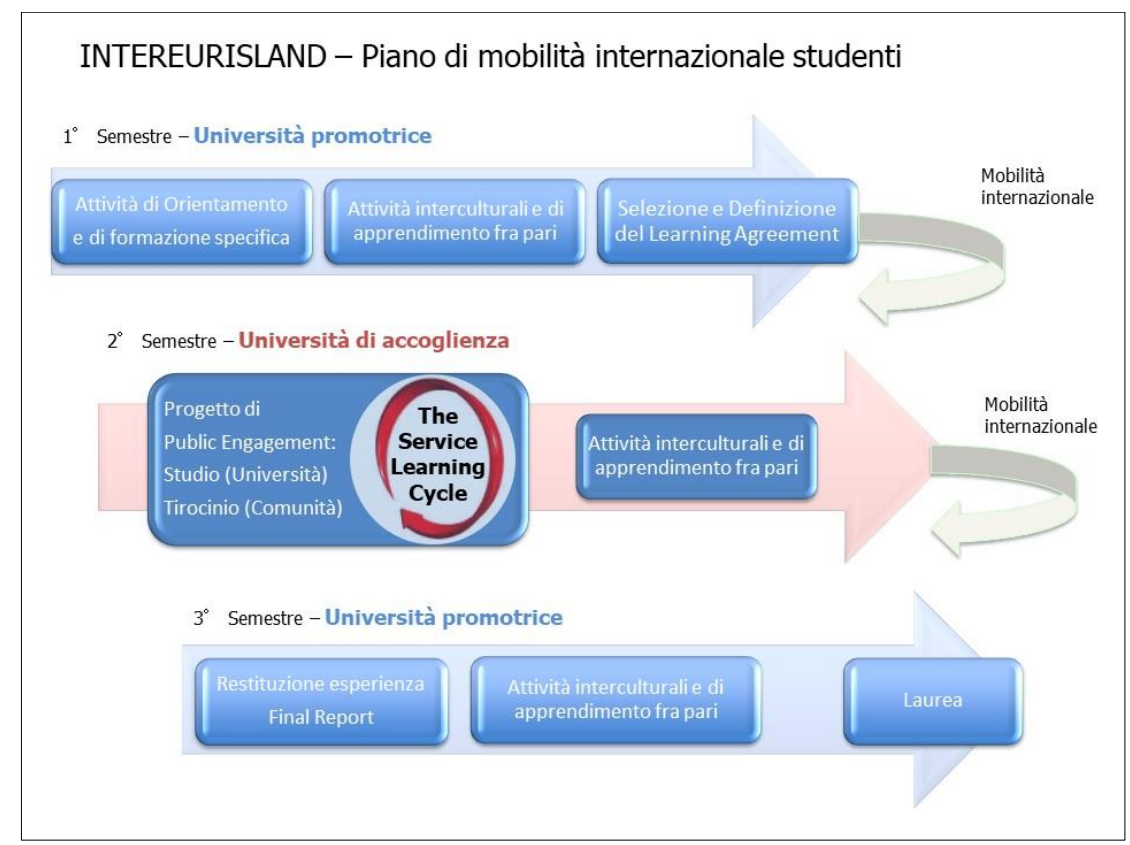

Fonte: Andrian (2018, pag.213)

Durante il periodo di mobilità in Brasile, nella logica della continua alternanza fra lo studio e la pratica, ho iniziato un percorso di formazione sulla comunicazione assertiva e sull'osservazione non giudicante grazie alle riunioni d'equipe, con il coordinatore e il tirocinante Maurizio Dorsa del Progetto $B E A^{79}$, svolte ogni quindici giorni, e grazie alla partecipazione al corso di 'Relazioni Interpersonali e dinamiche di gruppo' al DCH III /UNEB. Ho conosciuto questi due 'pilastri', su cui d'ora in poi continuerò a

\footnotetext{
${ }^{79}$ Un progetto sociale e di scambi interculturali promosso dalla Associazione EnARS di Padova (www.enars.it).
} 
lavorare, fondamentali sia nelle relazioni quotidiane ma soprattutto nelle relazioni che una psicologa instaura professionalmente. Ho iniziato ad imparare ad analizzare i miei vissuti, a spiegare cosa percepisco, quali emozioni provo e cosa posso imparare da queste sensazioni, in modo da poterlo applicare anche in futuro, scavando piano piano, anche ascoltando il mio corpo. Fondamentale è stata la stesura continuativa di un "diario" del mio tirocinio su cui ogni giorno scrivevo pensieri, esperienze, osservazioni e cose da ricordare del mio tempo passato nella istituzione che mi ha ospitata per il tirocinio.

Per quanto riguarda la parte pratica dell'esperienza di mobilità, le attività hanno seguito il Ciclo del Service Learning proposto dal National Youth Leadership Council e rielaborato da Andrian (2017) come in Figura.2.

Figura 2. Rielaborazione del Ciclo del Service Learning del NYLC

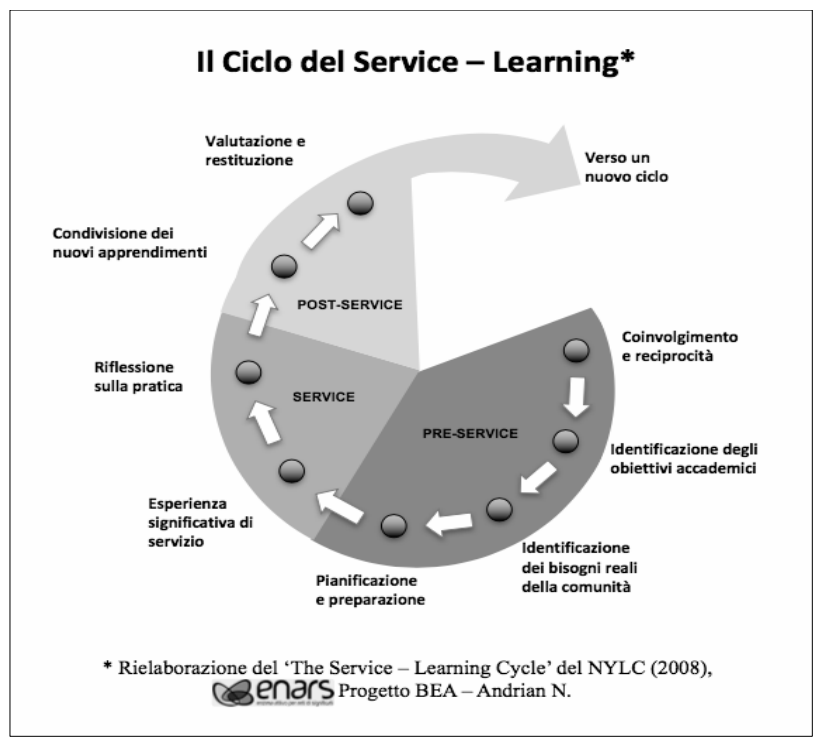

. Figura 2: reelaboração do Ciclo do Service Learning (EnARS - Progetto BEA)

Ho sviluppato il tirocinio nella struttura di semilibertà CASE NAENDA Gey Espinheira di Juazeiro-BA, che accoglie adolescenti in conflitto con la legge. Questa struttura nasce come casa rieducativa dove gli adolescenti passano tutta la settimana, partecipando ad attività interne ma anche esterne, come la scuola o il lavoro, avendo sempre orari di rientro precisi, ma con la possibilità di tornare a casa nel fine settimana grazie a una buona condotta.

Per quanto riguarda l'identificazione degli obiettivi accademici, prima di partire mi sono data degli obiettivi da inserire nel progetto formativo da presentare all'Università di Padova, coerenti anche con gli obiettivi di INTEREURISLAND: 
- Conoscere la cultura brasiliana nel contesto di Petrolina-PE e di Juazeiro-BA;

- Conoscere le attività proposte dell'Università dello Stato della Bahia/UNEB agli studenti stranieri;

- Approfondire e migliorare la mia conoscenza del Service-Learning dal punto di vista teorico e pratico;

- Partecipare al Progetto di responsabilità sociale (attraverso la metodologia del Service Learning) che il Dipartimento di Scienze Umane DCH III svilupperà con l'associazione CASE NAENDA Gey Espinheira (che accoglie adolescenti in conflitto con la legge);

- Conoscere la storia, la missione e come funziona l'associazione Case NAENDA Gey Espinheira;

- Conoscere le dinamiche di gruppo, formali e informali, tra gli adolescenti accolti nel Case Gey Espinheira, durante il mio tirocinio;

- Sviluppare e migliorare la mia conoscenza della relazione empatica dal punto di vista teorico (nel DCH III/UNEB) e pratico (nel CASE NAENDA Gey Espinheira);

- Sviluppare le mie abilità comunicative nella relazione con gli adolescenti del CASE NAENDA Gey Espinheira durante le attività che porterò avanti con loro;

- Approfondire la mia capacità di gestione delle mie emozioni nei momenti di stress e di difficoltà con gli adolescenti nel CASE NAENDA Gey Espinheira;

Per quanto riguarda la fase pratica di servizio, in un primo periodo ho osservato come l'equipe socio-psico-pedagogica dell'ente lavorava, imparando la loro routine e le varie regole. Mi sono subito ritrovata in un clima sereno nel quale mi sono sentita a mio agio. Tutta l'equipe si è dimostrata gentile e accogliente nei miei confronti. Poi, insieme alla psicologa Ivanessa Brito, abbiamo iniziato un percorso sul 'Progetto di Vita' per gli adolescenti, portato avanti nel momento di gruppo del giovedì pomeriggio.

Durante la preparazione delle attività abbiamo avuto la possibilità di confrontarci su vari argomenti e questi momenti sono stati fondamentali e di grande interesse per la mia crescita professionale. Per quanto riguarda la mia partecipazione, nello specifico, ho portato avanti un'attività sulle emozioni positive, un gruppo sul riconoscimento dei propri punti di forza, due attività ludiche e musicali, basate sulla costruzione di un flauto e di un tamburo e alla fine un'attività portata avanti individualmente, tramite colloquio per riflettere insieme a loro su cosa si porteranno a casa di positivo da questa esperienza di semilibertà. 
Piano piano ho iniziato a conoscere ciascuno di loro, ad imparare il loro modo di parlare e i loro simboli. A capire quando era il momento di scambiare due parole o scherzare e quando invece semplicemente sedermi vicino a loro in silenzio.

Tutti gli adolescenti mi hanno sempre trattata con rispetto, durante le attività di gruppo li osservavo e spesso sorridevo dentro di me nel porre attenzione a quanta cura riponevano in quello che facevano. In alcuni ho percepito questa pazienza e forza di volontà nel cercare di cambiare la propria situazione, studiando e cercando un lavoro serio.

Certamente non sono mai mancate le difficoltà, i momenti in cui non sapevo come reagire davanti a situazioni in cui mai avrei pensato di ritrovarmi, i momenti in cui mi sono fortemente preoccupata per alcuni di loro, date alcune vicende molto complesse, e i momenti in cui la diversità della lingua si faceva sentire in maniera frustrante. Ma il ritrovarmi lì ogni giorno ad osservare e imparare mi ha dato la spinta a voler conoscere di più e a perseverare nel mio percorso di formazione. Averlo fatto in una cultura diversa dalla mia mi ha ulteriormente dimostrato quanto sia importante la prospettiva da cui osserviamo le cose, quanto l'individuo sia il frutto di una ricchezza di elementi che noi futuri psicologi o psicoterapeuti dobbiamo sempre tenere in considerazione. Non possiamo tenerci i 'nostri occhiali' per incontrare e conoscere davvero l'altro.

Oltre ad aver avuto la possibilità di partecipare all'incontro con tutte le famiglie all'interno del CASE e alle visite settimanali del mercoledì pomeriggio, tutti gli adolescenti che ho incontrato mi hanno insegnato il valore e l'importanza della famiglia e della sua presenza, ad esempio quando il ricordo più bello della semilibertà diventava proprio lo stesso incontro con le famiglie sentendomi dire: "Non capita mai di essere tutti riuniti insieme e felici”. È stato da qui che è iniziato il mio pensiero sulla mia tesi di laurea 'Adolescenti in conflitto con la legge: analisi delle strategie familiari e interventi'. Nonostante i contesti familiari spesso conflittuali, rimangono fondamentali i rapporti di fiducia e di sostegno che l'adolescente crea con uno o più membri della famiglia, ad esempio la madre. È fondamentale dunque, rafforzare i legami con figure che possono essere una fonte di sostegno e svolgere un ruolo protettivo per lo sviluppo di questi adolescenti e per il cambio di traiettoria rispetto ai comportamenti di infrazione.

Oltre alla attività teoriche e pratiche descritte fino ad ora, il progetto INTEREURISLAND propone a noi studentesse e studenti in mobilità attività chiamate interculturali e de peer-education. A riguardo, non dimenticherò mai l'esperienza di essere stata facilitatrice del corso di lingua e cultura italiane che abbiamo tenuto al DCH III 
/'UNEB, anche in virtù della selezione dei nuovi studenti del corso di laurea in Pedagogia che, tramite il progetto, arriveranno in Italia nel 2020. Grazie a questo corso ho potuto presentare e insegnare la mia lingua e la mia cultura, apprezzando ancora di più le cose uniche che abbiamo in Italia ma anche la bellezza del confronto tra mondi diversi. Questa attività fa parte della formazione pre-partenza per le studentesse e gli studenti brasiliani che a è un punto fondamentale del progetto.

Alla fine del mese di Agosto ho avuto il privilegio di partecipare all'evento scientifico internazionale I Colóquìo Brasil Itália ${ }^{80}$, un momento di scambio e di incontro culturale (realizzato al DCH III / UNEB in Juazeiro) di grande importanza, data la presenza anche di una delegazione italiana con due docenti dell'Università di Padova, il prof. Massimo Santinello e il prof. Giuseppe Milan; le referenti/responsabili delle cooperative di Rovigo che, come partner italiane INTEREURISLAND, accolgono le studentesse e gli studenti brasiliani in mobilità (Roberta Lorenzetto e Beatrice Girotto); la presidente dell'EnARS, promotrice del Progetto BEA e dello stesso INTEREURISLAND (Isabella Polloni) e Nicola Andrian. Sono state giornate intense con incontri di ogni tipo tra la cultura italiana e quella brasiliana, in armonia verso un solo obiettivo: farsi promotrici di un'educazione contestualizzata come punto di partenza per nuove strategie di dialogo e incontro con l'altro, basato sulla logica dell'interculturalità, attraverso le nuove dimensioni globali. In questa occasione mi è stato chiesto di fare il mio primo intervento, in lingua portoghese, sull'esperienza che stavo portando avanti, insieme a Maurizio Dorsa e Jamile Soares da Silva, sia durante la presentazione dell'evento trasmessa via radio, grazie all'invito della radio locale "Sat Tropical", sia durante una sessione dell'evento stesso. Un altro evento importante a cui ho avuto la possibilità di partecipare è stato l'ENINEPE (“Encontro Integrado de Ensino, Pesquisa e Extensão da UNEB”) un incontro integrato sull'insegnamento, la ricerca e l'estensione dell'UNEB che si svolge annualmente a Salvador-BA. Questo evento vede ricercatori, coordinatori del centro di ricerca e post-lauream, direttori di dipartimento, insegnanti e studenti riunirsi con l'obiettivo di socializzare esperienze incentrate sulle opportunità di insegnamento, di ricerca e di responsabilità sociale dell'università, fornendo l'opportunità di discutere di etica all'università, attraverso conoscenza, innovazione e trasformazione. Anche qui ho partecipato a una presentazione sulla mia esperienza di interculturalità e ho

\footnotetext{
${ }^{80}$ Informazioni complete nel sito: www.coloquiobrasilitalia.com
} 
ascoltato diversi studenti brasiliani presentare la loro esperienza di studio, di tirocinio o di progetti di ricerca al di fuori del loro paese.

Nel pensare a delle possibili conclusioni di questo articolo, credo che almeno un'esperienza di interculturalità sia fondamentale da svolgere all'interno del percorso di formazione universitaria individuale e ringrazio il progetto l'EnARS, il progetto INTEREURISLAND e il DCH III UNEB per avermi dato la possibilità di vivere il tirocinio pre-laurea triennale in questo modo.

\section{Riferimenti bibliografici:}

ANDRIAN, N. INTEREURISLAND: from Field Research to a Possible Model for Internationalizing University Social Responsibility Experiences. In: BOFFO, V.; FEDELI, M. Employability \& Competences: innovative Curricula for New Professions. Firenze: Firenze University Press, pp. 205 - 219, 2018.

EnARS, «Progetto BEA - Educazione, formazione e intercultura - Un Ponte fra l'Italia e il Brasile», URL: http://www.enars.it/joomla/it/com-joomgalleryimagemanager/progetti/progetto-bea

ECA, lei no 8.069/90, Brasília, 13 de julho de 1990; $169^{\circ}$ da Independência e $102^{\circ}$ da República.

GOMES, Hugo Miguel dos Santos; PEREIRA, Maria Gouveia. Funcionamento familiar e delinquência juvenil: A mediação do autocontrolo. In: Análise Psicológica, 32(4), 439451, 2014.

NARDI, Fernanda Lüdke; DELL'AGLIO, Débora Dalbosco. Adolescentes em conflito com a lei: percepções sobre a família. In: Psicologia: Teoria e Pesquisa, 28(2), 181-192, 2012.

SISTEMA NACIONAL DE ATENDIMENTO SOCIOEDUCATIVO - SINASE/ Secretaria Especial dos Direitos Humanos - Brasília-DF: CONANDA, 2006.

SLAVET, J. D.; STEIN, L. A., KLEIN, J. L.; COLBY, S. M.; BARNETT, N. P.; MONTI, P. M.. Piloting the Family Check-Up With Incarcerated Adolescents and Their Parents. In: Psychological services, 2(2), 123-132, 2005.

TANGEMAN, Keegan; HALL, Susan. Self-Efficacy in Incarcerated Adolescents: The Role of Family and Social Supports. Child \& Youth Services. 32. 39-55, 2011.

\section{Sitografia}

www.intereurisland.blogspot.com

http://www.fundac.ba.gov.br/?page_id=2816 
www.portal.uneb.br/noticias/2019/08/09/juazeiro-coloquio-brasil-italia-destacaeducacao-contextualizada-e-estrategias-de-internacionalizacao www.portal.uneb.br/noticias/2019/10/18/eninepe-evento-reune-15-mil-professorespesquisadores-e-estudantes-em-cinco-dias-de-atividades

www.coloquiobrasilitalia.com 